\title{
Nonequilibrium transport and statistics of Schwinger pair production in Weyl semimetals
}

\author{
Szabolcs Vajna and Balázs Dóra \\ Department of Physics, BME-MTA Exotic Quantum Phases Research Group, Budapest University of Technology and Economics, \\ 1521 Budapest, Hungary
}

\author{
R. Moessner \\ Max-Planck-Institut für Physik komplexer Systeme, Nöthnitzer Strasse 38, 01187 Dresden, Germany
}

(Received 5 June 2015; published 13 August 2015)

\begin{abstract}
The nonequilibrium dynamics beyond the linear response of Weyl semimetals is studied after a sudden switching on of a dc electric field. The resulting current is a nonmonotonic function of time with an initial quick increase in polarization current followed by a power-law decay. Particle-hole creation à la Schwinger dominates for long times when the conduction current takes over the leading role with the total current increasing again. The conductivity estimated from a dynamical calculation within a generalized Drude picture agrees with the one obtained from Kubo's formula. The full distribution function of electron-hole pairs changes from Poissonian for short perturbations to a Gaussian in the long perturbation (Landau-Zener) regime. The vacuum persistence probability of high-energy physics manifests itself in a finite probability of no pair creation and no induced current at all times.
\end{abstract}

DOI: 10.1103/PhysRevB.92.085122

PACS number(s): 64.60.Ht, 05.70.Ln, 72.80.Ng, 37.10.Jk

\section{INTRODUCTION}

Condensed-matter systems, e.g., graphene, threedimensional (3D) topological insulators, and Weyl semimetals, provide a unique opportunity to examine fascinating QED effects, such as Klein tunneling, Zitterbewegung, chiral anomaly, or Schwinger pair production, most of which are barely accessible to experiment otherwise. In addition to this "fundamental" appeal, these phenomena play a crucial role in transport properties of these systems.

Weyl semimetals (WSMs) are 3D materials, which similar to the two-dimensional (2D) Dirac electrons in graphene, are characterized by linearly dispersing low-energy excitations around some points in the Brillouin zone [1-4]. These Weyl points are intersections of nondegenerate bands and are stable against perturbations according to their topological nature. The low-energy physics of these materials mimic the Weyl fermions well known from high-energy physics, giving the name WSM.

Similar to clean graphene, when the Fermi energy in WSMs is near the Weyl point, there are no charge carriers available for transport at zero temperature since the density of states vanishes as $\sim \epsilon^{2}$ close to the Weyl point. However, in an applied electric field, particle-hole pairs created by the Schwinger mechanism [5] contribute to transport.

The nonequilibrium state that evolves after turning on an electric field can be characterized by the statistics of the excitations and by the induced current. As pair creation is described by the Landau-Zener (LZ) formula in the strong electric-field regime, it is intrinsically related to the Kibble-Zurek (KZ) mechanism [6-8], which describes the universal scaling of defect generation in driven systems near a critical point. Alas, $\mathrm{KZ}$ scaling gives only the mean number of excitations and thus does not fully characterize the nonequilibrium state.

Such a characterization, however, is possible through all the higher moments or cumulants as these contain all information about nonlocal correlations of arbitrary order and entanglement. This is practically equivalent to determining the full distribution function of the quantity of interest. Therefore, the full distribution function of the number of electron-hole pairs is also of interest, yielding additional information about the physics of Schwinger pair production. Condensed-matter physics and cold atomic systems thus provide a unique way to experimentally detect such quantities $[9,10]$, beyond the current capabilities of high-energy physics. These ideas also relate to the discipline of full counting statistics [11,12] where outstanding experiments measure whole distribution functions $[13,14]$ and cumulants up to the 15 th order, e.g., in Ref. [15].

Our results on the time evolution of the current and statistics of electron-hole pairs is summarized in Table I. The time domain is split into three distinct regions with different behaviors, which we call classical (ultrashort), Kubo (short), and the Landau-Zener regime (long perturbations).

The time evolution of the current also allows us to conjecture qualitatively the behavior of the steady-state currentvoltage characteristics. For small voltages, the dynamical calculation combined with a generalized Drude theory reproduce the results of the Kubo formula calculations, i.e., the current is proportional to the electric field. However, Ohm's law breaks down for larger voltages, and the current-electric-field dependence becomes nonlinear. This critical electric field as well as the nonlinear current-voltage relation are important for possible transport experiments in WSMs.

The paper is structured as follows. First, we introduce the model and its solution in Sec. II. Then we discuss the evolution of the current and its implications for the steady-state conductivity in Secs. III and IV. The statistics of pair creation is studied in Sec. V, and it is compared with a complementary measure, the vacuum persistence probability, in Sec. VI.

\section{ELECTRIC-FIELD SWITCH ON IN A WEYL SEMIMETAL}

We consider noninteracting Weyl fermions near a single Weyl point. A homogeneous electric field is switched on at 
TABLE I. The electric field and time dependence of the total number of excitations or pairs created $(n)$ and their statistics, together with the electric current $(j)$ are shown. $\Lambda$ is the momentum cutoff, and $E$ is the electric field.

\begin{tabular}{lccc}
\hline \hline \multirow{2}{*}{ Time domain } & Classical & Kubo & Landau-Zener \\
& $t \ll \frac{\hbar}{v_{F} \Lambda}$ & $\frac{\hbar}{v_{F} \Lambda} \ll t \ll \sqrt{\frac{\hbar}{v_{F} e E}}$ & $\sqrt{\frac{\hbar}{v_{F} e E}} \ll t$ \\
\hline No. of pairs $(n)$ & $\sim E^{2} t^{2} \Lambda$ & $\sim E^{2} t$ & $\sim E^{2} t$ \\
Statistics & Poissonian & Poissonian & Gaussian-like \\
Current $(j)$ & $\sim E t \Lambda^{2}$ & $\sim E / t$ & $\sim E^{2} t$ \\
\hline \hline
\end{tabular}

$t=0$, which is described by a time-dependent vector potential $\mathbf{A}(t)=[e E t \Theta(t), 0,0]$. The time evolution of a given mode $\mathbf{p}=\left(p_{x}, p_{y}, p_{z}\right)$ is governed by the Hamiltonian,

$$
H=v_{F}[\mathbf{p}-e \mathbf{A}(t)] \cdot \boldsymbol{\sigma},
$$

where $\sigma$ denotes the vector of Pauli matrices and $v_{F}$ is the Fermi velocity. The spectrum consists of two bands as $\pm v_{F} \sqrt{p_{x}^{2}+p_{\perp}^{2}}$ with $p_{\perp}=\sqrt{p_{y}^{2}+p_{z}^{2}}$ as the perpendicular momentum. Initially $(t<0)$, the system is assumed to be in the $T=0$ vacuum state with all modes with negative single-particle energy filled and positive energy modes empty. This effective Weyl theory is valid at low energies compared to a high-energy cutoff $v_{F} \Lambda$ introduced for integrals over momentum space whenever necessary.

At $t=0$, the electric field is switched on, and the timedependent Schrödinger equation can be solved analytically using parabolic cylinder functions [16-18]. It is convenient to work in the adiabatic basis, which, following the derivation in Refs. [19,20], is achieved by a two-step unitary transformation $U=U_{s} U_{d}$. First, we apply a static rotation $U_{s}$ around the $x$ axis such that the new $y^{\prime}$ axis points in the direction of $\mathbf{p}_{\perp}=\left(0, p_{y}, p_{z}\right)$. Then we diagonalize the Hamiltonian with the dynamical $U_{d}=\exp \left[-i \delta(t) \sigma_{z} / 2\right]\left(\sigma_{x}+\sigma_{z}\right)$, where $\tan \delta(t)=\frac{p_{\perp}}{p_{x}-e E t}$. The time-dependent Schrödinger equation in the adiabatic basis reads as

$$
\begin{gathered}
i \hbar \partial_{t} \Phi_{\mathbf{p}}(t)=H^{\prime} \Phi_{\mathbf{p}}(t), \\
H^{\prime}=\sigma_{z} \epsilon_{\mathbf{p}}(t)-\sigma_{x} \frac{\hbar v_{F}^{2} e E p_{\perp}}{2 \epsilon_{\mathbf{p}}^{2}(t)},
\end{gathered}
$$

with the initial condition $\Phi_{\mathbf{p}}(0)=(0,1)^{T}$ corresponding to the fully occupied lower band. The wave function in the original basis is given by $U \Phi_{\mathbf{p}}$. The instantaneous eigenenergies form two bands as $\pm \epsilon_{\mathbf{p}}(t)$ with $\epsilon_{\mathbf{p}}(t)=v_{F} \sqrt{\left[p_{x}-e A(t)\right]^{2}+p_{\perp}^{2}}$. The pair creation is generated by the off-diagonal term $\sim \sigma_{x}$. By denoting the solution of Eq. (2) by $\Phi_{\mathbf{p}}(t)=\left[a_{\mathbf{p}}(t), b_{\mathbf{p}}(t)\right]$, the mode excitation probability $n_{\mathbf{p}}(t)=\left|a_{\mathbf{p}}\right|^{2}$, which gives the number of electrons created in the upper band due to the electric field and the holes in the lower band. The current operator in the original basis is $j_{x}=-e v_{F} \sigma_{x}$, which transforms into $j_{x}=-e v_{F}\left(\sigma_{z} \cos \delta+\sigma_{y} \sin \delta\right)$ in the adiabatic basis. This formula distinguishes between the conduction (intraband $\sim \sigma_{z}$ ) and the polarization (interband $\sim_{y}$ ) parts of the current. The current contribution $\left\langle j_{x}\right\rangle_{\mathbf{p}}(t)=j_{\mathbf{p}}^{\mathrm{c}}(t)+j_{\mathbf{p}}^{\mathrm{p}}(t)$ from a given mode $\mathbf{p}$ is determined by the mode excitation probability $n_{\mathbf{p}}(t)$ with the observation that $\operatorname{Re}\left\{i a b^{*}\right\}=-\frac{\epsilon_{\mathbf{p}}^{2}}{v_{F} p_{\perp} e E} \partial_{t} n_{\mathbf{p}}(t)[19,20]$,

$$
\begin{gathered}
j_{\mathbf{p}}^{\mathrm{c}}(t)=-e v_{F}\left[\frac{v_{F}\left(p_{x}-e E t\right)}{\epsilon_{\mathbf{p}}(t)}\left[2 n_{\mathbf{p}}(t)-1\right]\right], \\
j_{\mathbf{p}}^{\mathrm{p}}(t)=e v_{F} \frac{2 \epsilon_{\mathbf{p}}(t)}{v_{F} e E} \partial_{t} n_{\mathbf{p}}(t) .
\end{gathered}
$$

The total contribution of a Weyl node is obtained after momentum integration. In Eq. (4), the $n_{\mathbf{p}}$-independent background is discarded as an empty or fully occupied band does not carry current [19,21]. In our noninteracting model, the total current, excitation numbers, and higher cumulants are additive, i.e., given by the sum over the Weyl nodes.

The vanishing gap is a signature of the "criticality" of the WSM phase. As such, it exhibits scaling properties, which allow us to deduce important properties of the system without explicitly solving the Schrödinger equation. The excitation probability of the modes satisfies a scaling relation (in units of $\left.\hbar, v_{F}, e=1\right)$,

$$
n_{\mathbf{p}}^{E}(t)=n_{b \mathbf{p}}^{b^{2} E}\left(b^{-1} t\right),
$$

which follows from the time-dependent Schrödinger equation and holds for any choice of the dimensionless scaling parameter $b$. The invariants of the scaling transformation yield the natural dimensionless combinations which determine the physics, e.g., $\frac{p}{e E t}, \sqrt{\frac{v_{F}}{\hbar e E}} p, \tilde{t}=\sqrt{\frac{v_{F} e E}{\hbar}} t$, etc. The dimensionless time $\tilde{t}=\frac{t}{t_{E}}$ uniquely classifies the excitation probability as a function of $\mathbf{p}$, where $t_{E}=\sqrt{\hbar / v_{F} e E}$ is the time scale related to the electric field. Time-reversal considerations also give constraint on the excitation probabilities [18],

$$
n_{\mathbf{p}}(t)=n_{e \mathbf{E} t-\mathbf{p}}(t)
$$

which means that the excitation probability is symmetric with respect to $p_{x}=\frac{1}{2} e E t$, which is also apparent in the numerical solutions in Figs. 1 and 2. Accordingly, in Eq. (9) and everywhere where spherical coordinates are used, the momentum is measured from $(e E t / 2,0,0)$. That is, $p=$ $\sqrt{\left(p_{x}-e E t / 2\right)^{2}+p_{\perp}^{2}}$. At time $t$ momenta $\mathbf{p}$ and $e \mathbf{E} t-\mathbf{p}$ are related by the fact that they share the same ratio of level crossing in the Landau-Zener transition. For example in the particular cases of $p_{x}=0$ and $p_{x}=e E t$ there is only a
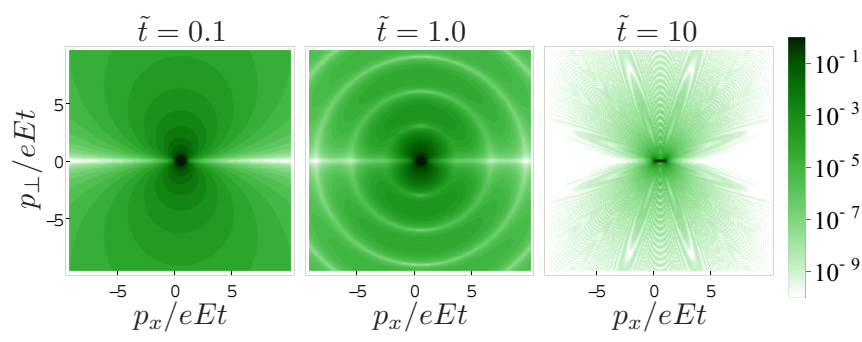

FIG. 1. (Color online) Excitation probabilities in momentum space for short (left), intermediate (middle), and long (right) perturbations. Although at short times $(\tilde{t} \ll 1)$ there are many excitations with large momenta, with increasing time the excitations are more and more confined to momenta $p \leqslant e E t$. Note the logarithmic scale on the color bar. 

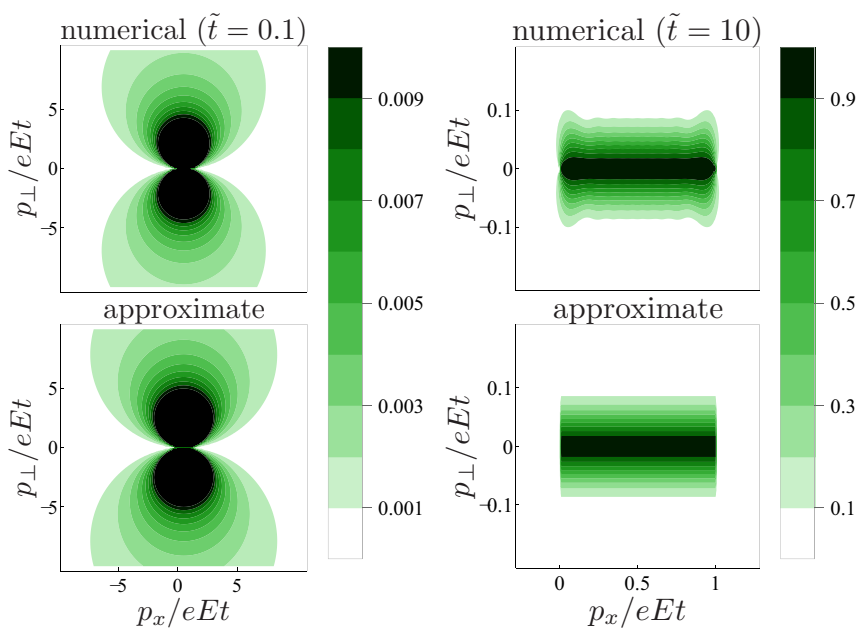

FIG. 2. (Color online) Comparison of the numerical and approximate excitation probabilities for short (left) and long perturbations (right). The excitation map has a "dipolar" character for short times, and the approximate formula (9) is nearly indistinguishable from the numerical solution for $p \gg e E t$. The excitation map is cylindrical for long times. An (asymptotically irrelevant) increased number of excitations at $p_{x}=0$ and $p_{x}=e E t$ is not captured in the approximation (10).

half-crossing [17], that is, the particles are not driven through the gap minimum, but the drive either starts or finishes there, respectively. To be more precise, the symmetry (7) originates in the following symmetry of the Hamilton operators: $H_{e \mathbf{E} t^{\prime}-\mathbf{p}}(t)=-H_{\mathbf{p}}\left(t^{\prime}-t\right)$, and $H_{e \mathbf{E} t^{\prime}-\mathbf{p}}^{\prime}(t)=H_{\mathbf{p}}^{\prime}\left(t^{\prime}-t\right)$ in the adiabatic basis. The time-evolution operator in the adiabatic basis is $\mathcal{U}_{\mathbf{p}}(t, 0)=\mathcal{T} \exp \left[-\frac{i}{\hbar} \int_{0}^{t} H_{\mathbf{p}}^{\prime}(s) d s\right]$, and applying the above symmetry transformation yields

$$
\begin{aligned}
\mathcal{U}_{e \mathbf{E} t-\mathbf{p}}(t, 0) & =\mathcal{A} \exp \left[-\frac{i}{\hbar} \int_{0}^{t} H_{\mathbf{p}}^{\prime}(s) d s\right] \\
& =\mathcal{K} \mathcal{U}_{\mathbf{p}}^{+}(t, 0) \mathcal{K},
\end{aligned}
$$

where $(\mathcal{A}) \mathcal{T}$ is the (anti-) time-ordering operator and $\mathcal{K}$ is complex conjugation. From this Eq. (7) follows.

The excitation probability as a function of $p$ is qualitatively different in the $\tilde{t} \gg 1$ and $\tilde{t} \ll 1$ cases (Figs. 1 and 2).

A perturbative solution valid for $\tilde{t} \ll 1$ is [19]

$$
n_{\mathbf{p}}=\frac{\left(e E \hbar p_{\perp}\right)^{2}}{4 v_{F}^{2} p^{6}} \sin ^{2}\left(\frac{v_{F} p t}{\hbar}\right) .
$$

This gives a good approximation for the excitation number for $p \gg e E t$. At short times high-energy states may become excited, which is reflected in the power-law decay of excitations as a function of momentum $\left(\sim p^{-2}\right.$ for $\left.p \ll \hbar / v_{F} t\right)$.

If the perturbation is long, the probability of exciting a given mode is well approximated by the LZ solution [22],

$$
n_{\mathbf{p}}=\Theta\left(p_{x}\right) \Theta\left(e E t-p_{x}\right) \exp \left(-\frac{\pi v_{F} p_{\perp}^{2}}{\hbar e E}\right) .
$$

This describes a "dynamical steady state," which is characterized by a longitudinally growing cylinder of excited states of length $e E t$ and radius $\sim \sqrt{\frac{\hbar e E}{\pi v_{F}}}$. In contrast to the short time limit, the excitation probability decays exponentially for large momentum. This exponential decay can be explained as a tunneling effect within the WKB approach [23].

Along with the analytical calculations, for comparison, we determine numerically $n_{\mathbf{p}}$ and $\partial_{t} n_{\mathbf{p}}$ by applying an explicit Runge-Kutta method [24] to solve the time-dependent Schrödinger equation. In Fig. 2 we compare the approximations used for $n_{\mathbf{p}}$ with the numerically obtained values.

\section{EVOLUTION OF THE CURRENT}

We are now in a position to discuss the time evolution of the current. The high-energy cutoff $v_{F} \Lambda$ defines an ultrashort time scale $t_{\Lambda}=\frac{\hbar}{v_{F} \Lambda}$, which satisfies $t_{\Lambda} \ll t_{E}$ for both condensed matter [2] and cold atomic [10] realizations of WSMs, similar to the $2 \mathrm{D}$ case [9]. The scaling property (6) implies a scaling for the integrated current as

$$
j_{E, \Lambda}^{\mathrm{c} / \mathrm{p}}(t)=b^{-3} j_{b^{2} E, b \Lambda}^{\mathrm{c} / \mathrm{p}}\left(b^{-1} t\right) .
$$

The particular choice of $b=t_{E}$ allows us to reveal the electric field and time dependence of the physical quantities. The current is expressed as $j_{E, \Lambda}^{\mathrm{c} / \mathrm{p}}(t)=E^{3 / 2} j_{1, t_{\Lambda} / t_{E}}^{\mathrm{c} / \mathrm{p}}\left(t / t_{E}\right)$. The scaling functions $j_{1, y}^{\mathrm{c} / \mathrm{p}}(x)$ are determined from Eqs. (4) and (5) after evaluating the momentum integrals with the particular form of $n_{\mathbf{p}}(t)$

$$
\begin{aligned}
j_{E, \Lambda}^{\mathrm{c}}(t) \sim E^{3 / 2} \begin{cases}-\left(\frac{t}{t_{E}}\right)^{3} \ln \frac{t t_{\Lambda}}{t_{E}^{2}}, & t \ll t_{\Lambda}, \\
-\left(\frac{t}{t_{E}}\right)^{3} \ln \frac{t}{t_{E}}, & t_{\Lambda} \ll t \ll t_{E}, \\
\frac{t}{t_{E}}, & t_{E} \ll t,\end{cases} \\
j_{E, \Lambda}^{\mathrm{p}}(t) \sim E^{3 / 2} \begin{cases}\frac{t t_{E}}{t_{\Lambda}^{2}}, & t \ll t_{\Lambda}, \\
(1+\text { nonuniv. }) \frac{t_{E}}{t}, & t_{\Lambda} \ll t \ll t_{E}, \\
\text { const, } & t_{E} \ll t .\end{cases}
\end{aligned}
$$

The term nonuniv. in the second line of Eq. (13) denotes the nonuniversal contribution from the high-energy regularization, which dies out with increasing time, as discussed further in Eq. (15).

For $t \ll t_{E}$ the current is dominated by the polarization part. Because of the large weight of high-energy states available to excite at ultrashort times $t<t_{\Lambda}$, the current is determined by the cutoff. The ultrashort time contribution of a Weyl point to the current is linear in time,

$$
j(t)=\frac{1}{6 \pi^{2}} \frac{e v_{F}}{\hbar^{3}} e E t \Lambda^{2} .
$$

This behavior has also been observed for 2D Dirac fermions [19] and can be explained using a classical picture of particles with effective mass $m_{i, j}^{-1}=\frac{\partial^{2} \epsilon_{\mathbf{p}}}{\partial p_{i} \partial p_{j}}$ accelerating in an external field satisfying Newton's equation. In 2D, the current saturates at $t \sim t_{\Lambda}$ and remains constant until $t \sim t_{E}$. In 3D the behavior is sharply different as the current starts to decay as $t^{-1}$ after reaching a maximal value at $t \sim t_{\Lambda}$. The precise form of the decay depends on the microscopic details (i.e., on the cutoff), but the exponent is a universal characteristic of Weyl physics. Imposing a sharp cutoff results in an oscillating current $j \sim t^{-1}\left[1+\cos \left(t / t_{\Lambda}\right)\right]$, also obtained within the linear response [25]. A smooth (exponential or Gaussian) cutoff of 


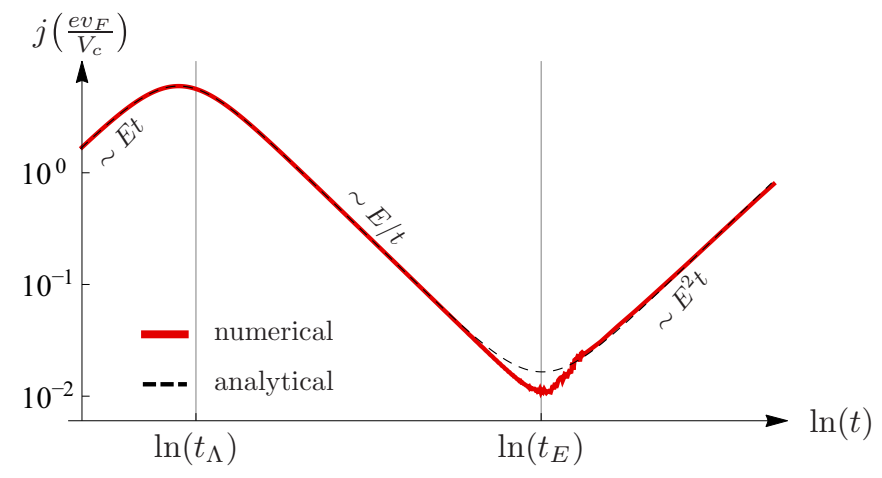

FIG. 3. (Color online) Time evolution of the total current after switching on an electric field. The analytical curve is the sum of polarization current (15), dominant for $t \ll t_{E}$, and conduction current (16), dominant for $t \gg t_{E}$. The evolution of the number of pairs $\kappa_{1}$ is shown in Fig. 4.

the form $\exp (-\sqrt{2} p / \Lambda)$ or $\exp \left(-p^{2} / \Lambda^{2}\right)$ does not generate the oscillating part and gives

$$
j(t)=\frac{1}{6 \pi^{2}} \frac{e^{2} E}{\hbar v_{F} t} F\left(t / t_{\Lambda}\right),
$$

where $F(x) \sim x^{2}$ for $x \ll 1$ and $F(x)=1 / 2$ for $x \gg 1$. The qualitative difference between the $2 \mathrm{D}$ and $3 \mathrm{D}$ cases is a consequence of their respective phase-space sizes. The polarization current is a sum of contributions with oscillating signs $j \sim \int d p \sin (2 p t) p^{d-3}$, which, by simple scaling, results in a time-independent contribution in 2D but decays as $t^{-1}$ in 3D.

The conduction part overtakes the polarization term at $t \sim$ $t_{E}$, beyond which the current increases linearly with time and nonlinearly with electric field as

$$
j(t)=\frac{1}{4 \pi^{3}} \frac{e^{3} E^{2}}{\hbar^{2}} t .
$$

This is simply the number of charge carriers per unit volume in the steady-state cylinder multiplied by $e v_{F}$. It is beyond the linear response as it depends quadratically on the external field [20]. Our analytical predictions for the current are illustrated in Fig. 3, together with the numerical results.

Bloch oscillations appear on a time scale $t_{\text {Bloch }} \sim \frac{\hbar}{e E a}$, where $a$ is the lattice constant, and our description holds for $t \ll t_{\text {Bloch }}$. The time scale related to the cutoff is nonuniversal, and both $t_{E}$ and $t_{\text {Bloch }}$ depend on the applied field. These three scales are in fact not independent, which can be seen in the following way. The momentum cutoff is proportional to the largest momentum in the system $\Lambda=\frac{1}{c} \frac{\hbar}{a}$, which relates the time scales as $t_{\Lambda} t_{\text {Bloch }}=c t_{E}^{2}$, where $c>1$ is a system-dependent constant describing the ratio of the linear size of the Brillouin zone and the validity range of Weyl physics. This also implies that in the experimentally relevant $t_{\Lambda} \ll t_{E}$ case, $t_{E} \ll t_{\text {Bloch }}$ is also satisfied, and all three regions appear before Bloch oscillations set in. $\Lambda$ is also limited by the separation of the closest Weyl points in the Brillouin zone. If two Weyl points lie close to each other, it limits the domain of applicability of our method through the parameter $c$. If $c \gg 1$, the Weyl physics describes only a small fraction of the Brillouin zone, and the contribution of the remaining parts can be large in the ultrashort perturbation limit. For intermediate and long perturbations, the cutoff does not play an important role, and the Weyl contribution dominates the total current and the excitation number. The results of a single Landau-Zener transition break down when the excitations are driven through another Weyl point. The time scale when it happens is given by $\frac{\Lambda}{e E}=\frac{1}{c} t_{\text {Bloch }}$ for an electric field pointing in the direction along which the two closest Weyl nodes are aligned, and it varies with the angle. To describe the behavior at $t \gg t_{\mathrm{Bloch}}$, one needs to go beyond the continuum description of Eq. (1) and consider the full lattice model as was performed for graphene in Ref. [26].

It is interesting to note that the maximal current is $j_{\max } \sim e^{2} v_{F} E \Lambda / \hbar^{3}$, which the system reaches upon leaving the classical region during the time evolution. Even in the nonlinear region in Eq. (16), the maximal current falls to the same order of magnitude, which is in sharp contrast to 2D Dirac semimetals where the nonlinear current strongly exceeds the current from the classical region.

As the external field induces current, it also injects energy into the system. The conduction and the polarization current decompose the total pumped energy into reversible (work) and irreversible ("heat") as follows. An infinitesimal change in the energy can be written as $d E=\sum_{i}\left(d \epsilon_{i} n_{i}+\epsilon_{i} d n_{i}\right)$, where $i=$ $(\mathbf{p}, \pm)$ runs over all momenta of the two bands. The first term corresponds to the reversible work performed on the system $d W=\sum_{\mathbf{p}} \partial_{t} \epsilon_{\mathbf{p}}\left(2 n_{\mathbf{p}}-1\right) d t=V E j^{\mathrm{c}}(t) d t$, whereas the second corresponds to the heat exchange $d Q=2 \sum_{\mathbf{p}} \epsilon_{\mathbf{p}} \partial_{t} n_{\mathbf{p}} d t=$ $V E j^{\mathrm{c}}(t) d t$ where we have expressed everything by the properties of the lower band. Correspondingly the work performed on the system and the heat are

$$
\begin{aligned}
& W=V E \int_{0}^{t} d s j^{\mathrm{c}}(s), \\
& Q=V E \int_{0}^{t} d s j^{\mathrm{p}}(s) .
\end{aligned}
$$

It is easy to check that the sum of the heat and work yields the total energy of the time-evolved state $\Delta E=W+Q=$ $\sum_{\mathbf{p}} 2 \epsilon_{\mathbf{p}} n_{\mathbf{p}}$, i.e., simply the sum of the energy absorbed by the excited modes.

\section{STEADY-STATE PICTURE FROM DRUDE THEORY}

The Drude picture provides a heuristic way to relate our results to optical conductivity studies of a WSM in the presence of impurities. In general, this is expected to work [21] for (contributions to) quantities independent of the relaxation time as, e.g., the high-frequency conductivity or the universal minimal conductivity of graphene [27]. In this spirit, the dynamics described above holds until a characteristic time determined by an effective scattering rate $1 / \tau$, and the zero-frequency limit of the ac conductivity can be estimated by substituting time as $t \rightarrow \tau$. This results in the counterintuitive conclusion that in the $t_{\Lambda}<\tau<t_{E}$ region, the conductivity is proportional to the scattering rate $\sigma(\omega \rightarrow 0) \approx \frac{e^{2}}{12 \pi^{2} \hbar v_{F} \tau}$, which agrees with the results of Ref. [28] based on Kubo formula calculations. Although this simple Drude picture works well for graphene [19], it fails to describe the transport properties of WSMs because in 3D, the density of states at the Weyl point vanishes even in the presence of small amounts 
of disorder [29], and concomitantly the quasiparticle lifetime diverges [2,3]. The Drude picture can be rescued if we apply it to $j_{\mathbf{p}}$, substituting the time variable with the momentumdependent lifetime and then evaluating the integral. The scattering rate in the Boltzmann or Born approximation is $1 / \tau_{\mathbf{p}}=2 \pi \gamma g\left(\epsilon_{\mathbf{p}}\right)[2,3]$, where $g(\epsilon)=\frac{\epsilon^{2}}{2 \pi^{2} \hbar^{2} v_{F}^{3}}$ is the density of states and $\gamma$ characterizes the scattering strength. In the large scattering limit $\gamma \gg \frac{\hbar v_{F}^{2}}{\Lambda}$, integrating Eq. (5) with $n_{\mathbf{p}}\left(\tau_{\mathbf{p}}\right)$ from (9) reproduces the results of Refs. [2,3], that is

$$
\sigma \sim \frac{e^{2} v_{F}^{2}}{\hbar \gamma}
$$

with a different prefactor and an additional logarithmic correction $\sim \frac{e^{2} v_{F}^{2}}{\hbar \gamma} \ln \left(\frac{\hbar v_{F}^{2}}{\gamma \Lambda}\right)$. The above treatment is valid for small electric fields $e E \ll \frac{\gamma^{2} \Lambda^{4}}{\hbar^{3} v_{F}^{3}}$ when the dominant contribution to the current comes from the momenta satisfying $\tau_{\mathbf{p}} \ll t_{E}$. In the case of additional Weyl nodes in the Brillouin zone, internode scattering can occur on a time scale $\tau_{\text {inter }}$. This also limits our real time study to $t<\tau_{\text {inter }}$, above which internode processes should be taken into account. This is clearly beyond the scope of the present paper.

If the scattering strength is small such that there is enough time for the modes to go through the LZ transition, then the steady-state occupation profile will be qualitatively similar to the LZ solution. As the quasiparticle lifetime is finite everywhere except in the close vicinity of the Weyl point, the cylinder of densely excited states will not extend to infinity but will be characterized by a finite length $e E \tau_{\text {eff }}(E)$. The precise form of $\tau_{\text {eff }}$ depends on the detailed nature of the scattering process. If there is a constant scattering rate $1 / \tau$, then $\tau_{\text {eff }}=\tau$, but generally it will depend on the electric field. The Drude picture estimates the stationary current in the nonlinear regime as

$$
j_{\text {stac }}=\frac{1}{4 \pi^{3}} \frac{e^{3} E^{2}}{\hbar^{2}} \tau_{\text {eff }}(E),
$$

and Ohm's law breaks down. The explicit $E$ dependence, however, depends strongly on the precise form of $\tau_{\text {eff }}(E)$. In case the relaxation time becomes independent of the electric field in the nonlinear region, a crossover from the $j \sim E$ linear region to a $j \sim E^{2}$ nonlinear region is expected.

\section{STATISTICS OF PAIR CREATION}

The expectation value and time evolution of the current is largely influenced by the number of pairs created as follows from Eqs. (4) and (5). This we now investigate in more detail. Although the expectation value of a quantity reveals much about underlying physical processes, fluctuations contain essential information as well and are important to provide a comprehensive description of the system [30]. Therefore, beyond simple expectation values, we study the fluctuations in the pairs created by their full distribution function. This provides a complementary measure to characterize the out-of-equilibrium state. As opposed to calculating the probability distribution function of pairs created directly, it is more convenient to work with the cumulant generating function (CGF) in unit volume, which is the logarithm of the characteristic function $\phi(\varphi)=\frac{1}{V} \ln \langle\exp (i \varphi \hat{N})\rangle$. Here, $\hat{N}$ denotes the excitation number operator, and the expectation value is taken with the time-evolved initial state. The CGF is expressed as a sum over momentum space,

$$
\phi(\varphi)=\frac{1}{V} \sum_{\mathbf{p}} \ln \left\{1+[\exp (i \varphi)-1] n_{\mathbf{p}}\right\} .
$$

The probability density function is the inverse Fourier transform of the characteristic function, that is, $p(n)=$ $\frac{1}{2 \pi} \int d \varphi \exp [V \phi(\varphi)-i n \varphi]$. For short perturbation, i.e., $t \ll$ $t_{E}$ the excitations add up from an extended region in momentum space with small excitation probability. The contribution from $p \lesssim 2 e E t$, where $n_{\mathbf{p}} \sim 1$, is negligible because of the small volume of the domain $\sim t^{3}$, and a Taylor expansion of the logarithm in Eq. (21) gives a good approximation

$\phi(\varphi)=[\exp (i \varphi)-1] \frac{1}{V} \sum n_{\mathbf{p}}$. That is, the total number of excitations per unit volume is Poissonian as $p(n)=$ $\lambda^{n} \exp (-\lambda) / n$ ! with

$$
\lambda=\frac{1}{12 \pi^{2}} \frac{(e E)^{2} t}{\hbar^{2} v_{F}} S_{2}\left(t / t_{\Lambda}\right),
$$

where $S_{2}(y)=\int_{0}^{y} d x \sin ^{2} x / x^{2}=y$ for $y \ll 1$, whereas it saturates to $\pi / 2$ for $y \gg 1$. All cumulants of the Poisson distribution are equal to the single parameter $\lambda$. The first cumulant is the expectation value, that is, for $t \ll t_{\Lambda}$ the excitations are created quadratically in time, whereas for $t_{\Lambda} \ll t \ll t_{E}$, the creation rate is constant. This behavior is clearly seen in Fig. 4 where we compare the numerically determined cumulants with the approximate solutions.

For $t_{E} \ll t$ the excited states are confined to a cylinder in momentum space, and substituting Eq. (10) into (21) yields

$$
\begin{gathered}
\phi(\varphi)=-\alpha \operatorname{Li}_{2}[1-\exp (i \varphi)], \\
\alpha=\frac{1}{8 \pi^{3}} \frac{(e E)^{2} t}{\hbar^{2} v_{F}},
\end{gathered}
$$

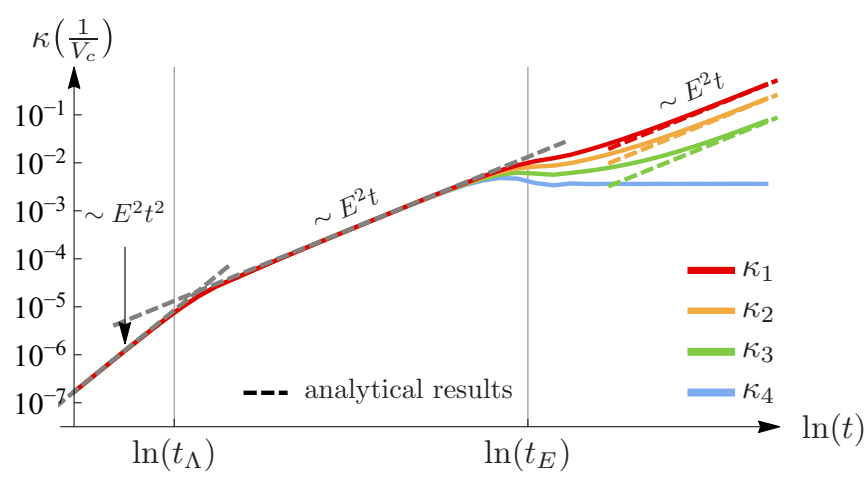

FIG. 4. (Color online) Time evolution of the cumulants $\left(\kappa_{1-4}\right)$ of electron-hole pairs per unit volume (log-log plot, numerical results). The cumulants coincide for $t \ll t_{E}$, which is a clear signature of a Poissonian distribution. The gray dashed lines show the $t \ll t_{\Lambda}$ and $t \gg t_{\Lambda}$ asymptotics of the analytical formula Eq. (22). For long times, the cumulants branch and follow the approximate formulas derived from Eq. (23) (colored dashed lines) within a time-independent constant coming from the difference between the exact $n_{\mathbf{p}}$ and the LZ approximation (Fig. 2). 
where $\operatorname{Li}_{2}(x)=\sum_{m=1}^{\infty} x^{m} / m^{2}$ is the dilogarithm function [31], in agreement with Ref. [32]. As time evolves the higher cumulants start to deviate from the first one, and the distribution is no longer Poissonian (see Fig. 4). The cumulants are determined from the series expansion of the CGF, the first few being $\kappa_{1}=\alpha, \kappa_{2}=\alpha / 2, \kappa_{3}=\alpha / 6$, and $\kappa_{4}=0$. Except for the variance all even cumulants vanish. There is a time-independent contribution from the $p_{x} \approx 0$ and $p_{x} \approx e E t$ regions in $n_{\mathbf{p}}$ (see Fig. 2), which is not captured in Eq. (10), which gets overwhelmed by the time-dependent terms. Apart from this, the cumulants listed above approximate very well the numerical results (Fig. 4). The peak of the distribution function is well captured in the central limit theorem (CLT) approximation, which states that the excitation number is Gaussian with mean $\alpha$ and variance $\sigma^{2}=\alpha / 2: p(n)=\frac{1}{\sqrt{2 \pi \alpha}} \exp \left\{-(n-\alpha)^{2} / \alpha\right\}$. This approximation neglects the cumulants higher than the second. The asymptotic decay of the distribution can be determined from the Gärtner-Ellis theorem [33], which in this case is essentially a saddle-point approximation of the inverse Fourier transform of the characteristic function. The probability of exciting a large number of pairs decays slower than estimated from the CLT but still in a Gaussian manner $p(n) \sim \exp \left\{-n^{2} / 2 \alpha\right\}$ (note the factor 2 difference in the denominator of the exponential with respect to the Gaussian distribution).

\section{PROBABILITY OF NO CURRENT AND THE VACUUM PERSISTENCE PROBABILITY}

Despite the applied electric field, there is a finite probability of no pair creation and no induced current, also known as the vacuum persistence probability. Analyzing the decay of this probability provides an alternative way to determine the pair-creation rate, which was used, e.g., by Schwinger in his seminal paper [5]. The vacuum persistence probability is $P_{0}(t)=|\langle\tilde{0}|U(t, 0)| 0\rangle|^{2}$, where $U(t, 0)$ is the time-evolution operator in the external field, $|0\rangle$ and $|\tilde{0}\rangle$ are the (Schrödinger) vacua at times 0 and $t$, respectively. With the knowledge of $n_{\mathbf{p}}$ it is expressed as

$$
P_{0}=\exp \left(-\sum_{\mathbf{p}} \ln \left(1-n_{\mathbf{p}}\right)\right) \equiv \exp (-V w t),
$$

where

$$
w=\frac{1}{t} \times \begin{cases}\lambda & \text { for } t \ll t_{E} \\ \frac{\alpha \pi^{2}}{6} & \text { for } t \gg t_{E}\end{cases}
$$

is the rate of vacuum decay per unit volume and time, increasing as $E^{2}$ and being independent of time for $t \gg t_{\Lambda}$ and increasing linearly with time for $t \ll t_{\Lambda}$. Alternatively, the pair-creation rate can also be defined as the total number of pairs created divided by the time it took, i.e., as $\kappa_{1} / t$. Nevertheless, these two definitions agree in the short-time limit and only differ by a constant prefactor in the long perturbation limit (Fig. 5). The vacuum persistence probability characterizes the time evolution similarly to the Loschmidt echo [34]: It measures the overlap of the nonequilibrium time-evolved wave function $U(t, 0)|0\rangle$ with a reference wave function, which in this case is the adiabatically evolved state.

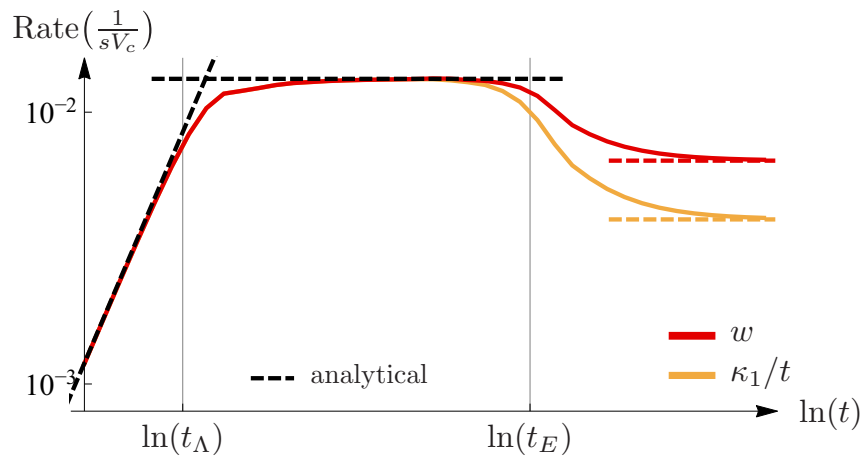

FIG. 5. (Color online) Particle-creation rate as a function of time estimated from the vacuum persistence probability and from the total number of excitations (log-log plot). The dashed lines show the results of Eqs. (22), (24), and (26).

So far we have assumed the initial state to be the ground state without any excitations, which describes the zero-temperature response of WSMs. An arbitrary initial distribution function can be handled similarly as long the modes with different momenta are independent, which is the case, e.g., at finite temperatures. Let $f(\mathbf{p})$ be the probability distribution function of having an excitation with momentum $\mathbf{p}$ in the initial state. The postquench occupation number is expressed as a weighted sum of the excitation probability of an unexcited and an excited mode as [18]

$$
n_{\mathbf{p}}^{f}=[1-f(\mathbf{p}-e \mathbf{E} t)] n_{\mathbf{p}}+f(\mathbf{p}-e \mathbf{E} t)\left[1-n_{\mathbf{p}}\right],
$$

where $f(\mathbf{p})=1 /\left[\exp \left(\beta \epsilon_{\mathbf{p}}\right)+1\right], \beta=1 / k_{B} T$. The initial number of excited states due to thermal fluctuations is $n_{T} \sim$ $1 /\left(\beta \hbar v_{F}\right)^{3}$, which is small near $T=0$, and does not modify qualitatively the results. This argument applies for systems with thermal initial density matrices, which are detached from the environment during time evolution. This assumption needs a thermalization time much longer than the observation time, which is usually not satisfied in condensed matter but could be achieved with cold atoms. Similarly a small deviation in the Fermi energy from the Weyl point gives only a subleading correction.

Our results can be readily applied also for inversion symmetric Dirac semimetals (referred to as structure I in Ref. [35]). The low-energy excitations of these systems are described by a $4 \times 4$ Dirac equation, which is decoupled to two degenerate Weyl fermions with opposite chirality. As the electric field does not couple these Weyl fermions, the two copies behave independently, which can be incorporated in a factor of 2 in the current and in the cumulants. On the other hand, in inversion symmetry-breaking Dirac semimetals (called structure II in Ref. [35]), the electric field couples the two copies of the (nondegenerate) Weyl fermions. If the inversion symmetry breaking is small, the results do not change qualitatively, but generally the description of the tunneling due to the electric field becomes more involved.

\section{CONCLUSION}

We have studied the nonlinear response of WSMs after switching on an external electric field before Bloch oscillations 
set in. The ultrashort time dynamics is nonuniversal, and the current depends on the details of the band structure at high energies. The current and the number of created electron-hole pairs grow linearly and quadratically, respectively, with time. The universal properties of Weyl nodes are manifested in the intermediate- and long-time responses. In particular, at intermediate times, the current decays as $1 / t$ due to the interplay of the number of created pairs and the available phase space. Particles are created at a constant rate, generating a Poissonian distribution for the statistics of the electron-hole pairs. At long times, the particle-creation rate takes on a constant value again, but the current starts to increase again in time because of the increasingly large number of pairs moving in the same direction. The distribution function of excitations crosses over from a Poissonian profile to a Gaussian distribution, which follows from the central limit theorem, applicable in the long-time limit due to the large number of pairs created.
The real time evolution of the current is translated to the conductivity of disordered WSMs within a generalized Drude picture, reproducing the results of previous calculations with different methods. This is a remarkable example of a problem from high-energy physics which naturally corresponds to one in condensed-matter physics with a separate set of observables, and which allows an exquisitely detailed analysis, thus holding the promise of a detailed experimental study in a tabletop experiment.

\section{ACKNOWLEDGMENTS}

This research has been supported by the Hungarian Scientific Research Funds No. K101244, No. K105149, and No. K108676, by the ERC Grant No. ERC-259374-Sylo, and by the Bolyai Program of the Hungarian Academy of Sciences.
[1] X. Wan, A. M. Turner, A. Vishwanath, and S. Y. Savrasov, Phys. Rev. B 83, 205101 (2011).

[2] A. A. Burkov and L. Balents, Phys. Rev. Lett. 107, 127205 (2011).

[3] P. Hosur, S. A. Parameswaran, and A. Vishwanath, Phys. Rev. Lett. 108, 046602 (2012)

[4] A. A. Burkov, J. Phys.: Condens. Matter 27, 113201 (2015).

[5] J. Schwinger, Phys. Rev. 82, 664 (1951).

[6] A. Polkovnikov, Phys. Rev. B 72, 161201 (2005).

[7] W. H. Zurek, U. Dorner, and P. Zoller, Phys. Rev. Lett. 95, 105701 (2005).

[8] B. Damski and W. H. Zurek, Phys. Rev. A 73, 063405 (2006).

[9] L. Tarruell, D. Greif, T. Uehlinger, G. Jotzu, and T. Esslinger, Nature (London) 483, 302 (2012).

[10] W.-Y. He, S. Zhang, and K. T. Law, arXiv:1501.02348.

[11] Y. V. Nazarov, Quantum Noise in Mesoscopic Physics (Springer, Berlin, 2003).

[12] M. Esposito, U. Harbola, and S. Mukamel, Rev. Mod. Phys. 81, 1665 (2009).

[13] V. F. Maisi, D. Kambly, C. Flindt, and J. P. Pekola, Phys. Rev. Lett. 112, 036801 (2014).

[14] N. Malossi, M. M. Valado, S. Scotto, P. Huillery, P. Pillet, D. Ciampini, E. Arimondo, and O. Morsch, Phys. Rev. Lett. 113, 023006 (2014).

[15] C. Flindt, C. Fricke, F. Hohls, T. Novotny, K. Netocny, T. Brandes, and R. J. Haug, Proc. Natl. Acad. Sci. USA 106, 10116 (2009).

[16] S. P. Gavrilov and D. M. Gitman, Phys. Rev. D 53, 7162 (1996).

[17] N. V. Vitanov and B. M. Garraway, Phys. Rev. A 53, 4288 (1996).
[18] N. Tanji, Ann. Phys. 324, 1691 (2009).

[19] B. Dóra and R. Moessner, Phys. Rev. B 81, 165431 (2010).

[20] B. Dóra and R. Moessner, Phys. Rev. B 83, 073403 (2011).

[21] N. W. Ashcroft and N. D. Mermin, Solid State Physics (Saunders College Publishing, Philadelphia, 1976).

[22] J. Hallin and P. Liljenberg, Phys. Rev. D 52, 1150 (1995).

[23] A. Casher, H. Neuberger, and S. Nussinov, Phys. Rev. D 20, 179 (1979).

[24] J. C. Butcher, Numerical Methods for Ordinary Differential Equations, 2nd ed. (Wiley, Hoboken, NJ, 2008).

[25] B. Rosenstein and M. Lewkowicz, Phys. Rev. B 88, 045108 (2013).

[26] M. Lewkowicz and B. Rosenstein, Phys. Rev. Lett. 102, 106802 (2009).

[27] A. H. Castro Neto, F. Guinea, N. M. R. Peres, K. S. Novoselov, and A. K. Geim, Rev. Mod. Phys. 81, 109 (2009).

[28] P. E. C. Ashby and J. P. Carbotte, Phys. Rev. B 89, 245121 (2014).

[29] Y. Ominato and M. Koshino, Phys. Rev. B 89, 054202 (2014).

[30] M. Campisi, P. Hänggi, and P. Talkner, Rev. Mod. Phys. 83, 771 (2011).

[31] I. S. Gradshteyn and I. M. Ryzhik, Table of Integrals, Series, and Products, 8th ed. (Academic, Waltham, MA, 2014).

[32] N. M. Vildanov, Phys. Rev. B 82, 033101 (2010).

[33] H. Touchette, Phys. Rep. 478, 1 (2009).

[34] A. Silva, Phys. Rev. Lett. 101, 120603 (2008).

[35] Z. Wang, H. Weng, Q. Wu, X. Dai, and Z. Fang, Phys. Rev. B 88, 125427 (2013). 